\title{
Radiographic patterns and associations of osteoarthritis of the hip
}

\author{
Joanna Ledingham, Stewart Dawson, Bryan Preston, George Milligan, Michael Doherty
}

\begin{abstract}
A number of patterns of osteoarthritis of the hip are described, though studies are conflicting with respect to the frequency of such patterns and their associations.

Two hundred and eleven patients (133 women, 78 men; mean age 66 years, range 29-86) referred to hospital with osteoarthritis of the hip were studied. Involvement was unilateral in $108(51 \%)$ and bilateral in 89 (42\%); $14(7 \%)$ had undergone arthroplasty and were presenting with osteoarthritis in their second hip (total 300 osteoarthritic hips). Sixty one per cent of hips had severe, $28 \%$ moderate, and $11 \%$ mild changes (Kellgren grade). Superior pole migration occurred in $82 \%$ (46\% superolateral, $25 \%$ intermediate, $11 \%$ superomedial), medial/axial migration occurred in only $8 \%$, and in $10 \%$ the pattern was indeterminate. In bilateral osteoarthritis of the hip the pattern was generally symmetrical. Superomedial and medial/axial patterns were proportionately more common in women, whereas superlateral osteoarthritis predominated in men. No association was found between multiple clinical nodes, radiographic polyarticular interphalangeal or first carpometacarpal osteoarthritis and any migration pattern. Any interphalangeal osteoarthritis was negatively associated with medial migration. Only $\mathbf{4 0} \%$ of hips could be categorised as hypertrophic or atrophic; chondrocalcinosis at any site was associated with atrophic osteoarthritis; no associations were seen with Forestier's disease.
\end{abstract}

This large survey confirms the association between chondrocalcinosis and atrophic osteoarthritis of the hip. Importantly it suggests that gender, rather than associated osteoarthritis at other sites, is a major determinant of the pattern of osteoarthritis of the hip.

(Ann Rheum Dis 1992; 51: 1111-1116)

The definition, description, and classification of osteoarthritis remain problematic. Although osteoarthritis of the hip is usually characterised predominantly in terms of radiographic appearance, differing aspects have been emphasised. For example, the severity of osteoarthritis can be estimated using a global method such as that described by Kellgren and Lawrence,' or by assessing separate features (e.g. osteophyte, narrowing) to give a summated or individual character score. ${ }^{2}$ Subclassification has been attempted mainly according to the pattern of migration of the femoral head within the acetabulum, ${ }^{3-7}$ or by the pattern of bone response to cartilage loss. ${ }^{7}$

The significance of these described patterns remains unclear, ${ }^{8-18}$ but associations with factors outside the hip have been described. For example, in some reports Heberden's nodes are associated with medial migration at the hip, ${ }^{89}$ and as medial and axial migration patterns typically occur in inflammatory arthropathies this has been taken as evidence to support an inflammatory aetiology for this generalised 'subset'. Similarly the presence of chondrocalcinosis at other joints has been associated with atrophic bone response and poor outcome at the hip, ${ }^{19-21}$ though mechanisms to explain this have not been proposed.

As a result of the marked heterogeneity of osteoarthritis differences in radiographic patterns at the hip could be useful in elucidating differences in aetiology and prognosis. This study was therefore undertaken to evaluate more fully the various radiographic patterns of osteoarthritis of the hip that have been described, namely the patterns of migration of the femoral head, patterns of bone response, and individual radiographic features of osteoarthritis of the hip, and to assess their associations with factors such as the presence of Heberden's nodes, interphalangeal osteoarthritis, multiple large joint osteoarthritis at other sites, and chondrocalcinosis. The relations of these factors to progression of osteoarthritis of the hip are addressed in a separate study.

\section{Patients and methods}

Over a two year period consecutive patients attending one rheumatology and three orthopaedic clinics (all general) with symptomatic osteoarthritis of the hip were recruited into the study. The diagnosis of osteoarthritis of the hip was defined as the presence of radiographic change (joint space narrowing and osteophyte, with or without cyst formation, sclerosis, or attrition) together with pain or clinical abnormalities, or both, at that site. Alternative arthropathies were excluded by clinical examination, blood tests (including erythrocyte sedimentation rate, serum calcium concentrations, rheumatoid factor (latex and RoseWaaler tests (titre $<1 / 64$ )), and absence of sacroiliitis, isolated osteonecrosis, and marginal erosions on radiographic screening. Aspiration of other affected joints (predominantly the knee) was undertaken to exclude gout and to confirm associated calcium pyrophosphate crystal deposition (compensated polarised light microscopy). 
Each patient underwent a full rheumatological history and examination with particular emphasis on the presence of activity, rest and night pain, duration of symptoms and past history of previous major trauma or surgery to the hips. On examination, clinical abnormalities consistent with osteoarthritis were noted for non-axial joints. Nodal change was defined clinically as the presence of typical superolateral Heberden's or Bouchard's nodes, or both, affecting at least three rays of each hand.

In each patient plain radiographs were taken of the pelvis (supine anteroposterior), hands (to include wrists), shoulders, knees (standing anteroposterior plus $20^{\circ}$ flexion lateral views), lumbar spine (anteroposterior and lateral), and thoracic spine (anteroposterior), together with any other clinically affected joint. Films were examined and scored by two observers during an initial training period. Subsequently one observer scored all hip films (observer 1) and the other all other films (observer 2). Both observers independently and blindly rescored 50 complete sets of radiographs selected at random. For the hips intra- (observer 1) and interobserver variability was determined by comparing these repeat gradings with the original assessment. Likewise for the other joint sites intra- (observer 2) and interobserver variability was also determined.

The Kellgren and Lawrence grading system ${ }^{1}$ was used to assess radiographic severity of osteoarthritis at the hips and other joints. Osteoarthritis was recorded as present in any joint scored as Kellgren grade 2 or more, and multiple large joint osteoarthritis was defined as at least one other large joint (knee, shoulder) affected in addition to the hip. Polyarticular interphalangeal osteoarthritis was defined by involvement (Kellgren score 2 or more) in at least three rays of both hands. Chondrocalcinosis and Forestier's disease (spinal hyperostosis with bridging new bone across four contiguous vertebrae $^{3}$ ) were recorded if present.

The pattern of migration of the femoral head within the acetabulum was classified into superolateral, superomedial, superior intermediate, medial or axial patterns according to

Table 1 Patient characteristics: radiographic osteoarthritis was defined as Kellgren grades 2-4

\begin{tabular}{|c|c|c|c|}
\hline Patient characteristic & Men & Women & $\begin{array}{l}\text { Odds ratios for } \\
\text { women } v \text { men } \\
\text { (95\% confidence } \\
\text { intervals) }\end{array}$ \\
\hline $\begin{array}{l}\text { Median (range) age (years) } \\
\text { Median (range) symptom duration (years) } \\
\text { Mean (range) ponderal index }\end{array}$ & $\begin{array}{l}65(29-84) \\
5 \cdot 5(1-36) \\
27 \cdot 1 \\
(20 \cdot 4-39 \cdot 7)\end{array}$ & $\begin{array}{l}68(42-86) \\
7(1-50) \\
26 \cdot 5 \\
(18 \cdot 2-47 \cdot 8)\end{array}$ & \\
\hline $\begin{array}{l}\text { No (\%) with osteoarthritis of the hip } \\
\text { Unilateral } \\
\text { Bilateral } \\
\text { Previous THR } \\
\text { No (\%) with isolated osteoarthritis of the hip } \\
\text { No (\%) with no radiographic osteoarthritis of }\end{array}$ & $\begin{array}{l}39(50) \\
37(47) \\
2(3) \\
29(37)\end{array}$ & $\begin{array}{l}69(52) \\
52(39) \\
12(9) \\
24(18)\end{array}$ & $\begin{array}{l}1.08(1.64 \text { to } 1.91) \\
0.71(0.40 \text { to } 0.80) \\
3.77(1.11 \text { to } 15.8) \\
0.37(0.20 \text { to } 0.69)\end{array}$ \\
\hline & $\begin{array}{l}35(45) \\
\text { r sites }\end{array}$ & $36(27)$ & $0.46(0.26$ to 0.82$)$ \\
\hline $\begin{array}{l}\text { Polyarticular interphalangeal joints } \\
\text { Any interphalangeal joint } \\
\text { First carpometacarpal joint } \\
\text { Knee joint } \\
\text { Glenohumeral joints } \\
\text { No }(\%) \text { with nodal changes } \\
\text { No }(\%) \text { with chondrocalcinosis }\end{array}$ & $\begin{array}{l}6(8) \\
30(38) \\
18(23) \\
18(23) \\
4(5) \\
6(8)\end{array}$ & $\begin{array}{l}24(18) \\
80(60) \\
53(40) \\
50(38) \\
17(13) \\
21(16)\end{array}$ & $\begin{array}{l}2 \cdot 60(6 \cdot 38 \text { to } 10 \cdot 6) \\
2 \cdot 42(1 \cdot 37 \text { to } 4 \cdot 26) \\
2 \cdot 21(1 \cdot 18 \text { to } 4 \cdot 13) \\
2 \cdot 01(1.07 \text { to } 3 \cdot 77) \\
1 \cdot 03(3.55 \text { to } 3 \cdot 76) \\
2 \cdot 25(1 \cdot 13 \text { to } 5 \cdot 72)\end{array}$ \\
\hline $\begin{array}{l}\text { Hip } \\
\text { Other sites } \\
\text { No (\%) with Forestier disease }\end{array}$ & $\begin{array}{l}2(3) \\
7(9) \\
9(12)\end{array}$ & $\begin{aligned} 0 & (0) \\
14 & (11) \\
4 & (3)\end{aligned}$ & $\begin{array}{l}1 \cdot 19(2.16 \text { to } 3.07) \\
0.24(0.08 \text { to } 0.74)\end{array}$ \\
\hline
\end{tabular}

the direction of movement of the femoral head. ${ }^{3}$ If migration could not be classified (due either to early osteoarthritis or severe attrition) the hip was categorised as indeterminate. The bone response at each hip was categorised as either atrophic or hypertrophic as described by Solomon $^{7}$; an indeterminate category was used for hips demonstrating a mixed pattern of bone response. In addition, individual features of osteoarthritis were graded 0-3 using a modification of the method of Thomas $e t a l^{2}$ at specific sites, namely narrowing (site of maximum narrowing only, maximum score 3), osteophyte (femoral neck, inferior and superior femoral head, medial and lateral acetabulum; maximum score 15), sclerosis (at site of maximum sclerosis; maximum score 3), cysts (acetabular roof, remainder of acetabulum, femoral head; maximum score 9), and attrition (femoral and acetabular components; maximum score 3 ).

Statistical analysis was performed using the $\chi^{2}$ (with Yates's correction) and Fisher's tests as appropriate. The $x$ statistic was used to assess intra- and interobserver variability ${ }^{22}$; multiple exclusive categories were used without the use of 'weights'. Odds ratios were calculated with respect to the presence or absence of putative risk factors.

\section{Results}

\section{PATIENT CHARACTERISATION}

Two hundred and eleven patients (133 women, 78 men; mean age 66 years, range 29-86) were recruited into the study (table 1 ). The mean age at onset of hip symptoms was 59 (range 16-84) years, giving a median symptom duration at the time of hospital referral of six (range 0.75-50) years. Fifty one per cent had unilateral and $43 \%$ had bilateral hip osteoarthritis; $6 \%$ had received a previous hip replacement and were presenting with their second hip affected. A total of 300 osteoarthritic hips was therefore assessed.

Sixty one per cent of hips had severe, $28 \%$ moderate, and $11 \%$ mild radiographic changes (corresponding to Kellgren grades 4, 3, and 2 respectively). Of the symptomatic hips $94 \%$ had definite radiographic osteoarthritis (Kellgren grade 2 or more (definite narrowing and other changes of osteoarthritis)) and 6\% (all of whom had symptoms and definite radiographic osteoarthritis in the contralateral hip) had Kellgren grade 0 or 1 . Sixteen per cent of hips showing definite radiographic osteoarthritis were asymptomatic; these included hips with marked attrition and similar severity of change as in the contralateral symptomatic hip. Twenty five per cent of patients had osteoarthritis confined to the hip; the remainder had either multiple large joint osteoarthritis (knees $32 \%$, shoulders $10 \%$ ) or hand osteoarthritis $(66 \%)$ with nodal changes occurring in $14 \%$. Women more commonly had nodal changes (table 1) and multiple joint involvement whereas men more commonly had isolated hip osteoarthritis (odds ratio 2.69; confidence interval $1 \cdot 36$ to $5 \cdot 35$ ).

PATTERNS OF MIGRATION

The patterns of migration of the femoral head within the acetabulum could be accurately 
Table 2 Migration and bone response patterns. Results given as number (\%) of patients

\begin{tabular}{lcccrr}
\hline & Men & Women & \multicolumn{2}{l}{ Age (years) } & \\
\cline { 4 - 6 } & & & $<5$ & $55-70$ & $71-90$ \\
\hline Migration & & & & & \\
$\quad$ Superolateral & $66(58)$ & $73(39)$ & $27(19)$ & $68(49)$ & $44(32)$ \\
Superior intermediate & $28(24)$ & $48(26)$ & $7(9)$ & $27(36)$ & $42(55)$ \\
Superomedial & $3(3)$ & $29(16)$ & $4(12)$ & $14(44)$ & $14(44)$ \\
Axial & $1(1)$ & $4(2)$ & $0(0)$ & $3(60)$ & $2(40)$ \\
Medial & $5(4)$ & $14(8)$ & $3(16)$ & $11(58)$ & $5(26)$ \\
$\quad$ Indeterminate & $12(10)$ & $17(9)$ & $4(14)$ & $11(38)$ & $14(48)$ \\
Bone response & & & & & \\
$\quad$ Atrophic & $20(17)$ & $37(20)$ & $6(10)$ & $22(39)$ & $29(51)$ \\
Hypertrophic & $23(20)$ & $38(20)$ & $12(19)$ & $34(56)$ & $15(25)$ \\
Indeterminate & $72(63)$ & $110(60)$ & $27(15)$ & $78(43)$ & $77(42)$ \\
\hline
\end{tabular}

categorised in $90 \%$ of affected hips (table 2, fig 1). Superior migration predominated, the superolateral pattern being the most common ( $46 \%$; fig 2$)$, followed by superior intermediate $(25 \%)$, and superomedial (11\%). The medial and axial patterns were infrequent, occurring in only 6 and $2 \%$ of hips respectively (fig 3 ). Patients with bilateral hip osteoarthritis generally showed symmetry in terms of the pattern of femoral head migration $(p<0.01)$; the axial pattern was only seen in those with bilateral osteoarthritis and, except for one patient, this was also true for the medial pattern. The exception (a 45 year old man who had undergone internal fixation of one femoral neck following a fracture at the age of 12) was superolateral migration in one hip with medial migration in the other (fig 4).

\section{PATTERNS OF BONE RESPONSE}

Bone response at the hip could be categorised as atrophic or hypertrophic in only $40 \%$ of patients. The atrophic response was seen in 19\%, hypertrophic in $21 \%$. No significant sex or age associations were noted for either pattern, though there was an increased frequency of the atrophic pattern with age.

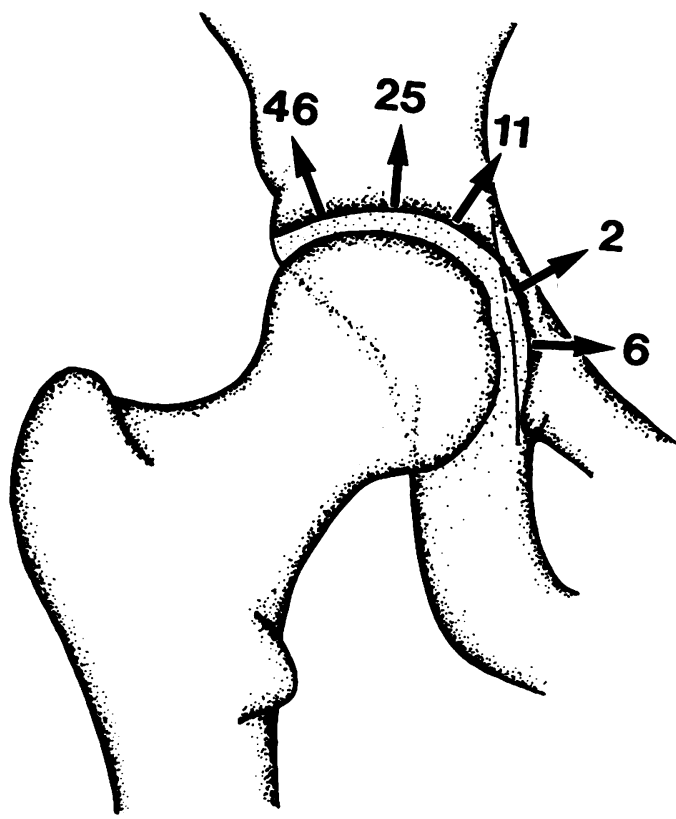

Figure 1. Diagram showing femoral head migration patterns in the 270 (90\%) hips that could be categorised.

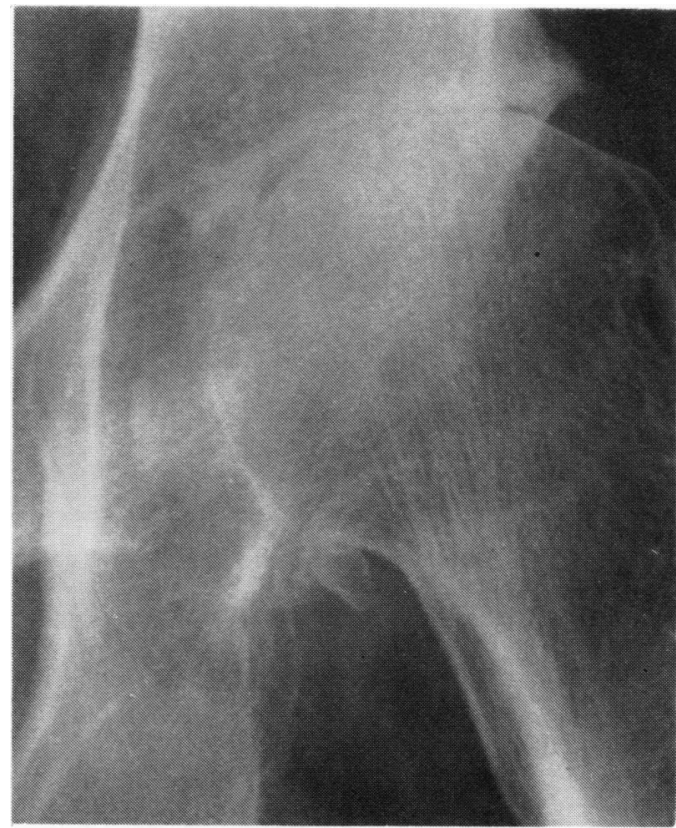

Figure 2 Radiograph showing superolateral femoral head migration.

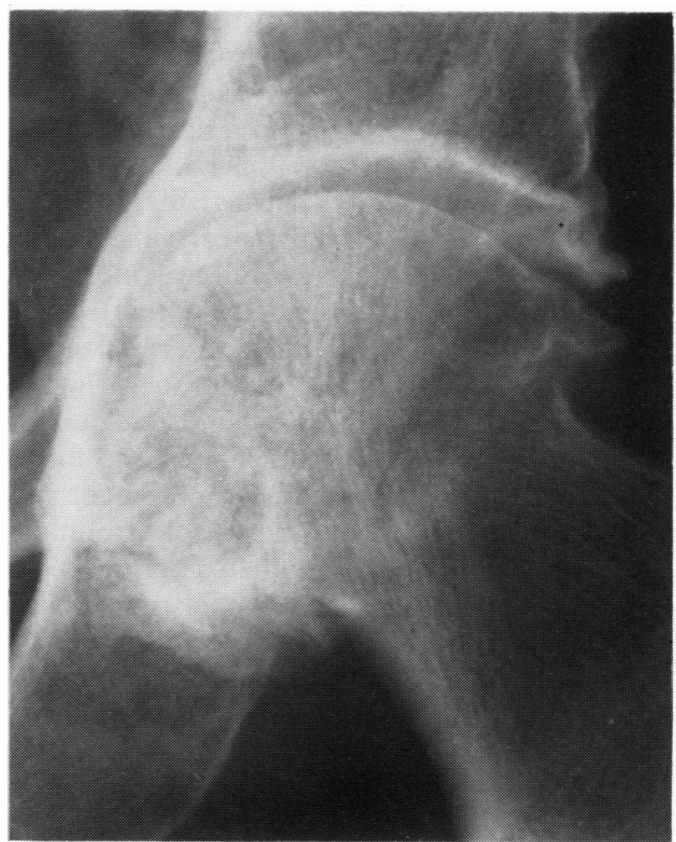

Figure 3 Radiograph showing the medial pattern of femoral head migration.

\section{INDIVIDUAL RADIOGRAPHIC FEATURES}

By definition mild, moderate, and complete joint space narrowing was seen in hips graded as Kellgren grade 2, 3, and 4 respectively. Marked osteophyte formation (score $>10$ ), cystic change (score $>6$ ), sclerosis (score $>2$ ), and attrition (score $>1$ ) were all seen exclusively in hips of Kellgren grade 4 . Complete absence of each of these radiographic features (in isolation or in combination), however, were seen in all of the Kellgren categories. Of the 18 symptomatic hips with no osteoarthritis on the Kellgren grading system (i.e. grades 0 and 1), 12 had mild osteophytosis, eight had mild cystic change, and three had mild sclerosis using the method of Thomas et al. ${ }^{2}$ Again by definition none of these hips had definite joint space narrowing. 


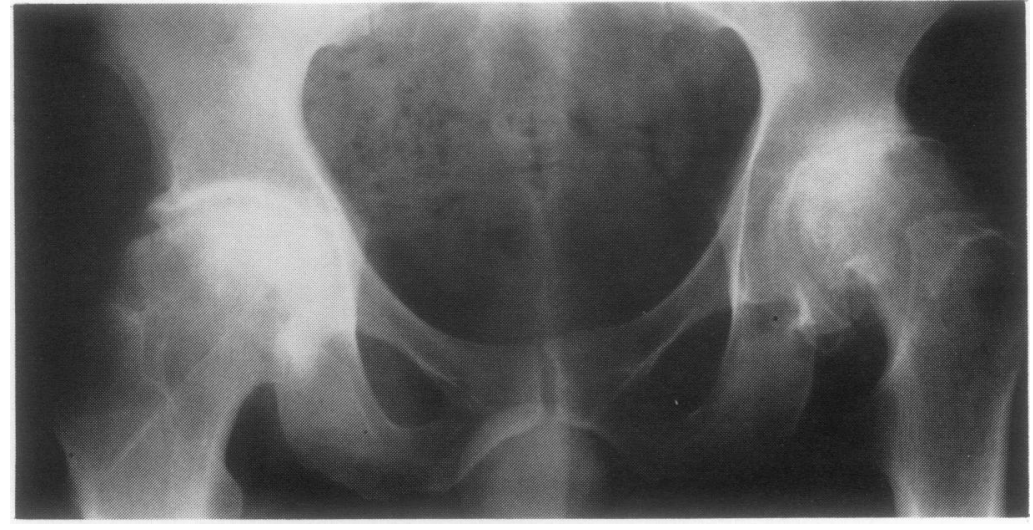

Figure 4 Radiograph of the only patient showing discordance in migration pattern with superolateral migration in the left and medial migration in the right hip (the latter having received mechanical insult and surgical intervention at the age of 12 years).

\section{ASSOCIATIONS}

Owing to the small numbers of hips showing medial or axial patterns of migration these two groups were merged for statistical analysis. Superomedial, medial, and axial patterns were proportionately more common in women $(26 \%$ of women $v 8 \%$ of men; $\mathrm{p}<0.001$ ) and the superolateral pattern was more common in men ( $58 \%$ of men $v 39 \%$ of women; $\mathrm{p}<0.01)$. No association was found between the migration pattern and age, the presence of nodal change, polyarticular interphalangeal osteoarthritis, first carpometacarpal joint osteoarthritis, or chondrocalcinosis. Medial and axial migration patterns were seen less frequently in patients with interphalangeal osteoarthritis compared with those without $(4 \% v 12.5 \% ; \mathrm{p}<0.05)$.

Chondrocalcinosis, either at the hip or at other sites, was more commonly associated with an atrophic bone response (occurring in 19, 3, and $9 \%$ of atrophic, hypertrophic, and indeterminate hips respectively; $\mathrm{p}<0.01$ ). There was no association between the type of bone response and the presence or absence of Forestier's disease, nodal change, first carpometacarpal joint osteoarthritis, polyarticular or pauciarticular interphalangeal osteoarthritis.

With regard to the individual radiographic features of osteoarthritis, bone attrition associated strongly with the atrophic bone response pattern and with reporting of night pain $(p<0.001)$. No significant associations were found between bone attrition, subchondral cyst or osteophyte formation, and the presence of nodal change, polyarticular or pauciarticular interphalangeal osteoarthritis, first carpometacarpal joint osteoarthritis, chondrocalcinosis or

Table $3 \times$ Values for observer variation

\begin{tabular}{lllll}
\hline & $\begin{array}{l}\text { Intra- } \\
\text { observer } \\
\text { variability }\end{array}$ & $\begin{array}{l}95 \% \\
\text { Confidence } \\
\text { interval }\end{array}$ & $\begin{array}{l}\text { Inter- } \\
\text { observer } \\
\text { variability }\end{array}$ & $\begin{array}{l}95 \% \\
\text { Confidence } \\
\text { interval }\end{array}$ \\
\hline Generalised nodal osteoarthritis & 0.78 & 0.19 to 1.37 & 0.63 & 0.01 to 1.26 \\
Interphalangeal osteoarthritis & 0.82 & 0.58 to 1.06 & 0.65 & 0.40 to 0.89 \\
Forestier disease & 0.58 & 0.26 to 1.42 & & 0.47 to 0.75 \\
Kellgren grade (hip) & 0.89 & 0.76 to 1.02 & 0.61 & 0.65 to 0.95 \\
Migration pattern & 0.88 & 0.74 to 1.02 & 0.80 & 0.28 to 0.81 \\
Bone response pattern & 0.73 & 0.49 to 0.97 & 0.55 & 0.41 to 0.75 \\
Hip attrition & 0.71 & 0.55 to 0.88 & 0.58 & 0.32 to 0.68 \\
Hip sclerosis & 0.57 & 0.40 to 0.73 & 0.50 & 0.28 to 0.56 \\
Hip osteophyte & 0.57 & 0.43 to 0.71 & 0.42 & 0.24 to 0.63 \\
Hip cyst & 0.54 & 0.44 to 0.64 & 0.44 & \\
\hline
\end{tabular}

Forestier's disease. A low total osteophyte score $(<6)$ associated strongly with the atrophic bone response pattern whereas a high score $(>9)$ associated with the hypertrophic pattern and was more commonly seen with superolateral migration.

The most frequent site for osteophyte, regardless of the migration pattern, was the superior femoral head. Although mild degrees of femoral neck osteophyte were seen in all migration patterns, moderate to florid osteophyte only occurred with superior patterns, most commonly with the superolateral $(68 \%)$ and least commonly with the superomedial $(10 \%)$. Osteophyte most often developed on the superior femoral head, femoral neck and lateral acetabulum with superolateral migration, and on the superior and inferior femoral head and neck with supermedial migration. Hips with medial and axial migration most often developed osteophyte on the femoral head with little osteophyte arising from the acetabulum or the femoral neck.

\section{REPRODUCIBILITY}

Table 3 gives the $x$ values for intra- and interobserver variability. Reproducibility was good for the pattern of femoral head migration, Kellgren grading of the hip, and to a lesser extent for the pattern of the bone response. Reproducibility was also good for assessment of osteoarthritis at joints other than the hip (range $x \quad 0.65$ for metacarpophalangeal joint osteoarthritis to 0.95 for knee osteoarthritis). Assessment of Forestier's disease was less reliable, as were assessments of individual radiographic features of osteoarthritis at the hip such as sclerosis, cyst, and osteophyte formation.

\section{Discussion}

There is no agreed method for radiographically assessing the severity or type of osteoarthritis of the hip. Several systems were therefore utilised in this large cross sectional study. Although the grading scale devised by Kellgren and Lawrence $^{1}$ is probably the most widely used method to assess severity it is primarily intended for epidemiological studies, places disproportionate emphasis on joint space narrowing and osteophyte (taking little account of features such as attrition and cyst formation), and assumes that the individual features of osteoarthritis show a linked and consistent pattern of progression. By contrast the grading system designed by Thomas $e t a l$, initially for use at the knee, ${ }^{2}$ assesses each individual radiographic feature of osteoarthritis. Though more complex and time consuming this system may allow more sensitive estimation of the process of osteoarthritis. In addition to these methods relating to 'severity', the pattern of migration of the femoral head ${ }^{3-6}$ and the pattern of bone response to cartilage $\operatorname{loss}^{7}$ have also been proposed as useful methods to categorise osteoarthritis of the hip.

We found the assessment of femoral head migration patterns easy to use and reliable, with good intra- and interobserver agreement. By 
contrast the pattern of bone response was more difficult to apply. Most hips did not fall into the specified categories of atrophic or hypertrophic, the high percentage of 'indeterminate' hips reflecting the common concurrence of atrophic and hypertrophic features in advanced osteoarthritis and difficulty in classifying hips with early or mild osteoarthritis. Good associations between the hypertrophic pattern and a high osteophyte score, and between the atrophic pattern and a low osteophyte score and presence of attrition, however, suggest that our assessments were consistent with the rationale proposed for division into these two extreme patterns. ${ }^{7}$ The grading of individual features using a modification of the system of Thomas $e t a l^{2}$ was easy to use but time consuming and proved less reproducible than the global grading system of Kellgren and Lawrence. ${ }^{1}$

The pattern of femoral head migration within the acetabulum has been assessed by a variety of investigators with some conflicting results regarding patterns and gender associations. ${ }^{3-6}$ In most studies the superior pole migration patterns predominate with the superolateral pattern in general being unilateral. ${ }^{3}$ We also found superior patterns to predominate with superolateral osteoarthritis clearly associating with male gender and female patients showing a stronger tendency to more medial patterns. Differences from other studies with regard to gender associations may in part relate to patient selection and study size. Previous reports relate to smaller numbers of patients principally recruited from orthopaedic clinics. Our study included a broad spectrum of rheumatology and orthopaedic clinic referrals, and allowed examination of 300 osteoarthritic hips. Nevertheless, it was still limited to symptomatic hospital referred patients and it is feasible that different findings could result from a community based study.

In accord with previous studies there was a low frequency of the medial and, in particular, the axial migration patterns. ${ }^{3-6}$ Unlike previous reports, however, we found no association between these patterns and nodal change. ${ }^{89}$ This difference may again relate to the larger size of this study or to definition in that we arbitrarily defined 'nodal' change clinically as the presence of Heberden's or Bouchard's nodes, or both, in at least three rays of each hand, rather than the presence of just one or a few nodes. Alternatively it is possible that the previously reported association between nodes and medial/axial hip patterns is indirect, reflecting the increased occurrence of both in women. The demonstrated negative association between medial and axial patterns and the presence of radiographic interphalangeal osteoarthritis (either polyarticular, widespread, or pauciarticular, limited) must seriously question any positive association between hand and hip osteoarthritis. Nodes and interphalangeal osteoarthritis may form different associations and are probably best considered separately for study purposes. Such discrepancies highlight the problems of definition and consistency between studies of osteoarthritis.

Symmetry of migration patterns in patients with bilateral hip osteoarthritis was striking and applied to all patterns. It has been suggested that medial and axial patterns, as they reportedly occur more commonly in both hips, are likely to result from a systematic disturbance whereas the superolateral pattern, reported to be more commonly unilateral, is likely to result from local mechanical factors (such as mild abnormalities of the contour of the femoral head or acetabulum $^{23}$ ). This study was not designed specifically to assess aetiology and the relative importance of systemic and local factors in the development and subsequent pattern of osteoarthritis at the hip remains unclear. The lack of association between nodes or radiographic interphalangeal osteoarthritis with migration patterns that classically occur in inflammatory arthropathies (medial/axial, reflecting more widespread rather than focal cartilage damage) must question the concept of a distinct subgroup of osteoarthritis resulting from a generalised inflammatory process and characterised clinically by nodal change. Although surprisingly little is known of sex differences in human hip anatomy it is established that the CE angle, ${ }^{24}$ a measure of acetabular depth, is larger in women. This could be a more reasonable explanation for the predominance of lateral migration in men and medial migration in women. The possibility that mechanical factors, resulting from gender differences in anatomy, are principal determinants of migration patterns in osteoarthritis of the hip is further supported by the one example of discordance in migration patterns that we encountered: a man with superolateral ('male') migration in one hip, but medial ('female') migration in the hip that had undergone surgical realignment (fig 4).

The distribution of osteophyte in the different migration patterns is also of interest with respect to mechanical determination. Hips with superolateral migration principally develop femoral neck 'buttressing' and osteophyte around the lateral joint margin, whereas hips with medial or axial migration develop osteophyte at the femoral head margins but little at the femoral neck or acetabulum. These findings support the concept that osteophyte formation is an active remodelling process that responds to altered biomechanical demands.

The association between chondrocalcinosis and atrophic hip osteoarthritis supports previous reports associating knee chondrocalcinosis with rapidly progressive osteoarthritis of the hip. ${ }^{19-21}$ This association, which remains unexplained, is particularly intriguing as calcium pyrophosphate crystal deposition at other sites such as the knee has been associated with hypertrophic radiographic changes at the site of deposition. ${ }^{25}$ Whatever the explanation this finding is contrary to the hypothesis that calcium pyrophosphate crystal deposition is a marker in subjects with osteoarthritis for a generalised (compared with local) tendency to a good bone response. ${ }^{25}$ No association was found between Forestier's disease and prominence of osteophyte or pattern of bone response.

The principal conclusions of this large study of osteoarthritis of the hip are that patient gender and local mechanical factors are impor- 
tant determinants of the pattern of femoral head migration. Although other constitutional or systemic factors may affect the pathogenesis of osteoarthritis of the hip the reported association between particular patterns of osteoarthritis of the hip and either nodal change or interphalangeal osteoarthritis is questioned.

J L is a Roussel (UK) Osteoarthritis Research Fellow. We are additionally grateful to Trent Regional Health Authority for financial support, and Professor A Wallace, Mr I W Forster, and $\mathrm{Mr} \mathrm{C} \mathrm{J} \mathrm{Howell} \mathrm{for} \mathrm{assistance} \mathrm{in} \mathrm{patient} \mathrm{recruitment.}$

1 Kellgren J H, Lawrence J S. Radiological assessment of osteoarthritis. Ann Rheum Dis 1957; 16: 494-502.

2 Thomas R H, Resnick D, Alazraki N P, Daniel D, Greenfield R. Compartmental evaluation of osteoarthritis of the knee. Radiology 1975; 116: 585-94.

3 Resnick D, Niwayama G. Degenerative disease at extra spinal locations. In: Resnick D, Niwayama G, eds Diagnosis of bone and joint disorders. Vol. 2. Philadelphia: Saunders, 1981: 1322-35.

4 Resnick D. Patterns of migration of the femoral head in osteoarthritis of the hip. Roentgenographic-pathologic osteoarthritis of the hip. Roentgenographic-pathologic Correlation and comparison with

5 Gofton J P. Studies in osteoarthritis of the hip: part 1. Classification. Can Med Assoc $\mathcal{F} 1971 ; 104: 679-83$.

6 Hayward I, Bjorkengren A G, Pathria M N, Zlatkin M B, Sartoris D J, Resnick D. Patterns of femoral head migration in osteoarthritis of the hip: a reappraisal with CT and pathologic correlation. Radiology 1988; 166: 857-60.

7 Solomon L. Patterns of osteoarthritis of the hip. $\mathcal{F}$ Bone foint Surg [Br] 1976; 58: 176-83.

8 Marks J S, Stewart I M, Hardinge K. Primary osteoarthritis of the hip and Heberden's nodes. Ann Rheum Dis 1979; 38 107-11.

9 McGoldrick F, O'Brien T. Osteoarthritis of the hip and Heberden's nodes. Ann Rheum Dis 1989; 48: 53-5.
10 Meachim G, Whitehouse G H, Pedley R B, Nichol F E Owen R. An investigation of radiological, clinical an pathological correlations in osteoarthritis of the hip. Clin Radiol 1980; 31: 565-74.

11 Jeffery A K. Osteophytes and the osteoarthritic femoral head. f Bone foint Surg [Br] 1975; 57: 314-24.

12 Resnick D. Osteophytes of the femoral head and neck. Arthritis Rheum 1983; 26: 908-13.

13 Solomon L. Geographical and anatomical patterns of osteoarthritis. $\mathrm{Br} \mathcal{F}$ Rheumatol 1984; 23: 177-80.

14 Goften J P. A classification of osteoarthritis of the hip and its relevance to pathogenesis. $\mathcal{F}$ Rheumatol $1983 ; 10$ (suppl 9): 65-6.

15 Hermodsson I. Roentgen appearance of coxarthrosis Relationship between the anatomy, pathologic changes, and roentgen appearance. Acta Orthop Scand 1970; 41: 169-87.

16 Cameron H U, Macnab I. Observations on osteoarthritis of the hip joint. Clin Orthop Rel Res 1975; 108: 31-40.

17 Macys J R, Bullough P G, Wilson P D. Coxarthrosis: a stud of the natural history based on a correlation of clinical radiographic, and pathologic findings. Semin Arthritis Rheum 1980; 10: 66-78.

18 Harrison M H M, Schajowicz F, Trueta J. Osteoarthritis of the hip: a study of the nature and evolution of the disease. f Bone foint Surg [Br] 1953; 35: 598-626.

19 Menkes C J, Decraemere W, Postel M, Forest M. Chondrocalcinosis and rapid destruction of the hip. $\mathcal{F}$ Rheumatol 1985; 12: 130-3.

20 Menkes C J, Simon F, Delrieu F, Forest M, Delbarre F. Destructive arthropathy in chondrocalcinosis articularis. Destructive arthropathy in chon

21 Gerster J C, Vischer T L, Fallet G H. Destructive arthropathy in generalised osteoarthritis with articular chondrocalcinosis. F Rheumatol 1975; 2: 265-9.

22 Cohen J. A coefficient of agreement for nominal scales. Education E Psychological Measurement 1960; 20: 37-46.

23 Murray R O. The aetiology of primary osteoarthritis of the hip. Brf Radiol 1965; 38: 810-24.

24 Resnick D, Niwayama G. Anatomy of individual joints. In: Resnick D, Niwayama G, eds. Diagnosis of bone and joint disorders. Vol. 1. Philadelphia: Saunders, 1981: 107-12.

25 Doherty $M$, Dieppe $P$ A. Clinical aspects of calcium pyrosphosphate dihydrate crystal deposition. Rheum Dis Clin North Am 1988;14: 395-414. 